\title{
QResonancias

\section{El Pensamiento Latinoamericano como CONCIENCIA Y ASUNCIÓN DE DOS MUNDOS Posibles Discusiones desde la Filosofía de la Historia}

\author{
The Latin American Thought as \\ AWARENESS AND ASSUMPTION OF TWO WORLDS \\ Possible ARguments FROM the PHILOSOPHY OF History \\ FECHA DE RECEPCIÓN: 30/08/2016 - FECHA DE ACEPTACIÓN: 28/11/2016
}

\author{
Diego Pereira Ríos ${ }^{1}$ \\ pereira.arje@gmail.com \\ Universidad de Montevideo
}

Resumen

\begin{abstract}
Si existe o no un pensamiento latinoamericano sigue siendo una discusión necesaria entre los pensadores actuales. Creemos que es una necesidad que Latinoamérica sea hoy el sujeto de su propio filosofar. Con la ayuda de algunos filósofos examinamos la propuesta de Andrés Belaúnde y su síntesis viviente, donde el elemento existente en estas tierras asume el elemento externo para luego fusionarse en una nueva condición. Y también tomaremos la propuesta de Leopoldo Zea que nos habla de un proyecto asuntivo donde debemos asumir lo que somos desde una yuxtaposición de culturas que se conjugan resultando ser los que somos: mezcla de europeo y de latinoamericano. Desde estos elementos, y como un caso a examinar con valor histórico y de actualidad, proponemos una mirada desde el ejemplo del pueblo guaraní del Chaco boliviano, a partir de algunos pensadores actuales.
\end{abstract}

Palabras clave: filosofía de la historia, asunción, síntesis viviente, proyecto asuntivo, sujeto latinoamericano.

\begin{abstract}
Whether there is or not a Latin-American thought continues to be a necessary discussion today. We believe that is necessary to Latin America to be the subject of its own philosophizing. With the help of some philosophers, we exanimate the proposal of Andrés Belaúnde and his concept of living synthesis, where the existing element on these land takes the external element to merge later in a new condition. We will also take the proposal of Leopoldo Zea, which talks us about an assumptive project, where we must assume what we are from the perspective of a juxtaposition of cultures that conjugates and has as a result being what we are: mix of European and Latin American. From these elements and as a case to examine its historical value and topical importance, we propose an account from the Guarani people in the Bolivian Chaco and from some contemporary thinkers.
\end{abstract}

Keywords: philosophy of history, assumption, living synthesis, assumptive project, latin amierican sujet.

\footnotetext{
${ }^{1}$ Estudiante del Profesorado en Filosofía, Universidad de Montevideo. Ha publicado recientemente el libro La fuerza de la esperanza. Montevideo: Nueva Visión Editorial, 2016.
} 
Hablar hoy de una filosofía de la historia va más allá de un problema filosófico, es la exigencia propia de pensar la historia a partir de la filosofía. Esta necesidad tiene la característica de intentar volverse sobre sí misma, de re-flexionarse a partir de la búsqueda en su pasado histórico para analizar su presente, examinarlo, reinterpretarlo intentando comprenderse y encontrar en él un telos, un fin o una finalidad que le dé sentido. Al hablar del sentido a partir de la filosofía de la historia nos referimos al para qué de los acontecimientos históricos, lo cual hace de la filosofía un saber que parte de ciertos supuestos (los hechos) pero que se encaminan a pronunciar juicios sobre el futuro, claramente difíciles de afirmar como verdaderos.

Este saber que intenta la filosofía tendrá por tanto un carácter profético confirmado por autores como Pieper: "Una filosofía de la historia debe ser capaz, además, de ser profética: profeta es el que habla del futuro de un modo que es apropiado únicamente al pasado, o que habla sobre un presente a la luz del futuro tratado como un hecho consumado". (cit. en Benavides, 1994: 33). Ésta es una condición necesaria de una filosofía de la historia que, en este trabajo, pretende encontrar en la historia del pensamiento latinoamericano, ciertas pistas que den cuenta del presente. La historia latinoamericana ha sido marcada por un discurso que intentó ser hegemónico, contado desde afuera, pero desde hace varios años tenemos acceso a un conocimiento que nace de la misma experiencia latinoamericana, y por ello creemos que nos acerca a una cierta verdad a partir de la realidad interna de la historia.

\section{La historia latinoamericana como sujeto de la filosofía.}

Leopoldo Zea, en la investigación acerca del pensamiento latinoamericano, afirma que el hombre americano "es un hombre montado entre dos mundos: el que deja y, que al dejar, ya no le pertenece; y uno nuevo mundo, con su propia historia, pero con una historia que no siente suya, que tampoco le pertenece" (1969: 62). Por ello, si pretendemos hacer filosofía de la historia latinoamericana, debemos partir desde esta gran disyunción que ha recorrido la reflexión de los pensadores latinoamericanos y que nos fue llevando a colocarnos en el centro, para poder hacer filosofía. Arturo Ardao dirá que es lo americano como objeto de la filosofía, como materia a examinar y estudiar, lo cual no alcanza para comprender la filosofía particular de la historia de un pueblo.

Como sujetos históricos estamos implicados en el legado que recibimos del pasado y por ello debemos partir del nosotros para lograr una filosofía original. Era necesario que aflorara el ser americano, pues como dirá Ardao: "la americanidad de la que habría 
de ser, o ya era, nuestra filosofía, radicaba implícitamente, antes en la personalidad de su sujeto que la naturaleza de su objeto" (1987: 83). Era necesaria la independencia antropológica y ontológica del ser americano para que trajera consigo una independencia intelectual. El hombre americano necesitaba reconocerse como sujeto, persona activa y consciente de sí mismo, para lograr comprenderse desde el interior de su propia historia y dar sentido a los acontecimientos vividos y que fueron conformando su propio ser.

Cuando hablamos del sujeto americano nos referimos a un sujeto plural, un sujeto social y comunitario con una cierta continuidad histórica, en la cual se va construyendo y reconociendo a sí mismo como valioso. El sujeto americano ha buscado desde siempre una unidad coherente para poder relatar su propia historia y no sólo ser objeto de estudios extranjeros. Por ello le era necesario encontrar rasgos y características propias que lo diferencien como un nosotros: un sujeto que unificara el sentir y pensar de los habitantes de nuestra tierra. Pero este nosotros era inalcanzable desde fuera. Era necesaria la formulación desde el interior mismo del ser americano.

La historia latinoamericana necesita entonces de un sujeto que se apropie de su pasado y de su presente logrando así ser un sujeto activo. De ahí que se fue forjando un nosotros latinoamericano que va descubriendo lo que le pertenece como propio. Dice Arturo Roig:

El punto de partida para una definición de lo nuestro y del nosotros ha de ser siempre el sujeto concreto inserto en su mundo de relaciones humanas desde el cual recibe o se apropia de las formas culturales, y no lo recibido de sí mismo, cuya riqueza intrínseca se juega toda entera en el acto de recepción (2009: 63).

Por ello, junto a la necesidad de reconocerse, también se nos hace imprescindible el elemento externo para lograr una mejor comprensión de lo que somos y de lo que es lo nuestro.

A este proceso por el cual vamos adquiriendo elementos culturales desde afuera, le debemos sumar los componentes propios de nuestro ser latinoamericano y es desde ellos que debemos construir nuestra historia. La historia latinoamericana es así el sujeto de la filosofía porque es pensada por los mismos latinoamericanos que comienzan a desarrollar una filosofía marcada por una crisis de identidad que se embarca en la búsqueda de su propia personalidad. Este proceso irá variando según los mismos actores del pensamiento y por ello unos rechazarán la idea de una identidad propia latinoamericana mientras otros rechazarán toda influencia externa para defender una 
postura y un pensamiento propio.

\section{La toma de conciencia latinoamericana.}

La historia de Latinoamérica es generalmente conocida desde el discurso hegemónico del conquistador colocando siempre a América como el objeto, siendo el sujeto activo, el europeo en un primer momento, y el estadounidense después. En las diversas posturas ante este hecho creemos que, si deseamos hacer de la historia latinoamericana el sujeto de la filosofía, debemos pensarla desde el mismo interior que la atraviesa y que nos atraviesa. No podemos evitar nuestro origen ya que esto potencia nuestro ser y lo hace aún más complejo y más rico. Somos esta mezcla de lo que fuimos y de lo que se nos agrega del que era antes de nosotros y que llegó a estas tierras.

La ciencia intenta explicar el mundo a través de la relación de causas y leyes de la naturaleza, pero no logra comprender las consecuencias o resultados de los hechos. Y menos aún lo podría hacer si hablamos de hechos sociales compuestos por sujetos. Es aquí donde hay un gran quiebre en la comprensión del hombre europeo acerca de la existencia de un ser latinoamericano, pues no puede hacer experiencia de ello. Es por eso que la historia como relato propio rescatará un conocimiento que escapa a la ciencia sin que sea, por ello, menos valioso. Se hizo necesaria una historia propia pues "una historia representa una forma peculiar de recoger, organizar y transmitir información, que debe valorarse con criterios específicos y a la que hay que dirigir las preguntas que le son pertinentes" (Cruz, 2008: 192). La historia relatada por los mismos actores que viven la experiencia nos acerca a un grado de conocimiento más profundo y de mayor alcance.

Por ello en la construcción del relato histórico el mismo sujeto se va comprendiendo a sí mismo como protagonista y se descubre junto a otros. Es entonces que la subjetividad lleva a una intersubjetividad: del "yo" vamos desembocando en un "nosotros". No se trata de una sola experiencia sino de nuestra experiencia, y por ello es la experiencia de "lo nuestro", de nuestro ser latinoamericano (Roig). Es el sujeto plural que comienza a ser consciente de la necesidad de pensarse a sí mismo en el mundo. Pregunta Enrique Dussel: “¿en qué consiste esa historia de la filosofía de latinoamericana? Pienso que es una hermenéutica histórica, una interpretación como autoconciencia de la propia historia en su sentido de acontecimiento" (1992: 208).

Esta toma de conciencia no fue algo fácil de lograr. Latinoamérica -como lo afirma Zea- se vio en la tensión de estar entre dos mundos: el del colonizador y el del coloni- 
zado, y estos mundos se fueron construyendo de diferente forma en nuestra tierra hasta el momento de encontrarse y entremezclarse. Hay una confrontación de ambos mundos, de dos paradigmas y cosmovisiones diferentes, en la cual históricamente se vieron en oposición y que nos colocó en una situación de crisis. El proceso por el cual se fue logrando la toma de conciencia lo entendemos en términos de una transición.

El término "transición" propuesto por Karl Löwith alude al pasaje de cierto estado a otro diferente, sin perder en ello su esencia. En este sentido la conciencia histórica del hombre puede cambiar y trascender los momentos históricos, pero sin perder en ello su propio ser. Por ello en el encuentro de los dos mundos, el europeo y el latinoamericano, si bien se alcanzan cambios críticos profundos, hay un elemento que pertenece a ambos grupos que los asemeja y produce un proceso de progreso humano hacia algo nuevo. Dice Löwith:

si supusiéramos de un cambio histórico en la esencia humana, dicho cambio sólo podría producirse porque el ser humano se mantiene esencialmente igual en todos los avatares, pues sólo lo que se mantiene puede cambiar. De lo contrario no podríamos reconocer como tal aquello que se ha modificado y cambiado (1998: 241).

Incluso esa transición se da en medio de la crisis mundial, donde el hombre occidental se enfrentaba a la crisis de la modernidad que luego será expresada por el existencialismo. Esta es la posibilidad que el hombre latinoamericano descubra una filosofía propia a partir del hombre que va naciendo en esta tierra. Nos propone Zea que la nueva filosofía

justificará las aspiraciones que se hacían ya expresas en el pensamiento latinoamericano, un pensamiento que los latinoamericanos temían llamar filosofía, enajenados como estaban con la idea de que sólo en las expresiones del hombre occidental y su cultura se hacía expresa la universalidad a la que, se suponía, debería aspirar toda filosofía (1969: 77).

Por eso esta transición se va desarrollando en medio de un sentimiento de inferioridad del hombre latinoamericano ante el hombre europeo, de modo que, al salir el hombre europeo del lugar de modelo, el hombre latinoamericano comienza a pensarse y a confiar en sí mismo.

Siguiendo a Roig, dentro de este proceso de transición hay un momento de ruptura, de quiebre, que no lo podemos identificar en un momento exacto, sino que es parte del mismo proceso, pero que posibilita la toma de conciencia del hombre latinoamericano. 
Esta experiencia de ruptura tiene que ver con la aceptación de lo que somos en determinado momento y la percepción que hay sobre ello, pues no son lo mismo. Para ello hay que superar varias dificultades: comprender que la universalidad del fenómeno de ruptura que se da en toda sociedad que busque su identidad; que la importancia que se le da a los conceptos de "raza latina" o "raza española" no indican ninguna diferencia en el orden del ser; tampoco hay diferencias a nivel de las conciencias por más que los hombres en cuestión tengan diferente origen. Por otro lado también habrá diferencias a nivel discursivo que dependerán de las ideologías involucradas que no denotan la misma realidad. Así tendremos la polaridad barbarie-civilización, continente en brutocontinente del espíritu, entre otros.

Roig habla entonces de dos conciencias de ruptura: una conciencia inocente, que tiene que ver con quien padece las situaciones de forma pasiva, como víctima, desde la expatriación de su propia tierra, conformando una masa de pueblos y campesinos desplazados. Esta conciencia inocente tiene que ver con los que perciben su situación de inferioridad al europeo, pero no son los responsables de la permanencia dentro de ella. Distinta es la conciencia culposa. Dentro de ella están los sujetos que la viven pero con cierto grado de responsabilidad, justificando su situación de diversas maneras. Esta misma conciencia culposa es la que fundamenta hasta hoy las sociedades dependientes, en la cual se elaboran argumentos en defensa de sus intereses, dejando de lado los intereses más generales de la población. Pero en ambos casos dice Roig que se trata: "de un sujeto que se encuentra imposibilitado de ejercer una autoafirmación de sí mismo como valioso y de entenderse como una natura naturans, es decir, como actor de su hacerse y gestarse" (2009: 281).

Como resultado de la conjunción de estas dos conciencias surge en Latinoamérica la conciencia de un hombre nuevo que asumió el sentimiento del conquistador y el sentimiento de orgullo del hombre de estas tierras: el mestizo. Rodó afirma que es el hombre mestizo el que se encuentra en una profunda actitud de ruptura frente a dos tradiciones: la ajena extranjera, a la que no pertenece, y a la que lo antecede geográfica e históricamente, pero a la cual no pertenece. Esta conciencia es la que atravesará el tiempo y las diferentes mentalidades latinoamericanas y es la que los pensadores intentarán conjugar en un sólo pensamiento. La conciencia culposa es el rasgo común de este hombre ya que viven en sí mismo, el conquistador dominador, y el conquistado dominado. 


\section{La "síntesis viviente” de Víctor A. Belaúnde.}

Víctor Andrés Belaúnde (Arequipa 1883 - New York 1966) fue un jurista, intelectual, destacado orador y político, filósofo de la cultura que hará muchos aportes a la sociología peruana, integraría la "generación del novecientos" una de las generaciones más articuladas a nivel intelectual que siguiendo la obra de Rodó, fueron denominados arielistas. Estudió Derecho pero desde muy temprano mostró dedicación a la filosofía. Considerado agnóstico en su juventud, Belaúnde vuelve a la fe católica alcanzando a ser un destacado apologista cristiano. Tiene varias obras de importancia, pero nuestro trabajo se centrará sobre todo en su obra La síntesis viviente, escrita en 1950 en España, donde tenemos acceso a su filosofía de la cultura.

Frente al problema del hombre latinoamericano dirá Belaúnde que este intercambio de culturas, que se entrecruzan en un determinado tiempo y espacio por la acción de una sobre la otra, se desarrolla como un proceso de síntesis viviente, entendiendo por síntesis, por un lado, el resultado de la conjunción de una cultura que es la asumente (la conquistadora) y de la segunda: la asumida (o conquistada); y por otro lado síntesis refiere a la encarnación de ciertos valores espirituales pero que se encarnan materialmente en elementos históricos. Es clara la distancia que tomará Belaúnde tanto de Hegel como de Marx al rechazar todo idealismo y todo materialismo, pues no podemos reducir a uno u otro este encuentro de valores, solamente.

Si bien el estudio Belaúnde lo centra en la historia y sociedad peruanas, la piensa en términos de toda Latinoamérica. Él "defiende una interpretación de la cultura como diversidad integrada y orgánica y de explicar su evolución por determinantes espirituales" (Belaúnde, 1950: 211). Salazar Bondi confirmará esta afirmación central de la síntesis o asunción de Belaúnde: es a través de los valores espirituales que podemos conjugar los elementos de la cultura que aparentan oponerse o distanciarse. Belaúnde afirmará: "hay en cada cultura una forma o esencia que está constituida por valores superiores, y hay una materia compleja que es la psicología de cada nación, influida por la herencia, por el factor geográfico y por la estructura económica" (1950: VII-VIII).

Pero veamos como presenta Belaúnde la explicación de esta interacción de los valores:

Los valores espirituales asumen y transforman los elementos que constituyen la corporeidad de una nación: tierra, instituciones, estructuras, quedan penetradas y transidas por los mismos principios e ideales. Éstos realizan penosamente, a través del tiempo, una obra de inspiración, de impregnación y de asunción (1950: VIII) 
En este sentido podemos afirmar que las culturas que habitaban en estas tierras, desde el momento que son asumidas por la cultura del conquistador, se van transformando en una nueva cultura que perderá algunos elementos que se verán transidos por elementos propios de hombre español. Y aún más, propone una mirada nueva sobre este mismo hecho: no sólo se daría una imposición desde arriba hacia abajo de valores extranjeros sobre los autóctonos, sino que hay un proceso de "encarnación": hay un contenido espiritual que desciende y toma forma nueva en el elemento material. No se sustituyen unos por otros, sino que son transformados sin desaparecer.

Según Podetti no podemos asignar las categorías de materia y forma aristotélicas a los elementos de la síntesis de Belaúnde (donde la materia sería el elemento indígena y la forma el elemento español) pues al pensar en el mestizaje como posible resultante de la mezcla, no corresponde totalmente a la tesis fusionista, ya que si bien se puede aceptar esto en el orden material (biológico), no es tan aceptable en el término de los valores espirituales ${ }^{2}$. Si se aceptara solamente esta síntesis en el orden material caeríamos en un materialismo dialéctico a la manera de Marx que "deduce que las condiciones de vida material de las sociedades son las que determinan las ideas, las opiniones colectivas y las instituciones" (Juan Carlos Zaffaroni, 1966: 21).

La propuesta de síntesis de Belaúnde se distanciará también de la dialéctica hegeliana. Según Hegel la realidad es fundamentalmente devenir a través de sus tres momentos de tesis, antítesis y síntesis, a través de las cuales se avanza paso a paso y por ello también el pensamiento sigue este mismo curso ${ }^{3}$. En este movimiento eterno del espíritu absoluto se dan las oposiciones de la idea y el mundo material para alcanzar la síntesis en el hombre, pero

no se trata de una simple conciliación, sino que la tesis lleva necesariamente a la antítesis, y viceversa, y este movimiento del ser conduce inexorablemente a la síntesis, en la cual se encuentran conservadas y superadas la tesis y la antítesis. Y cada estadio encuentra su verdad en el siguiente (Marías, 1979: 311).

Esto lleva a Hegel a afirmar que en el proceso histórico cada movimiento dialéctico implica que en la conquista de un pueblo sobre otro sólo se mantienen las características del primero.

Belaúnde no compartirá que sean lo mismo la tesis-antítesis hegeliana que su propuesta del proceso asuntivo a través del binomio asumente-asumido:

\footnotetext{
${ }^{2}$ Podetri, J. Ramiro, Cultura y alteridad: En torno al sentido de la experiencia latinoamericana, Ed. Monte Ávila Editores, Caracas, 2007, p. 178

${ }^{3}$ Cfr. Brugger, W., Diccionario de Filosofía, Ed.Herder, Barcelona, 1962, p.146
} 
La historia de la civilización es un proceso de síntesis vivientes que se integran y se superan. En todas ellas hay un elemento asumente y otro asumido. Los elementos asumidos no pierden su esencia, sino que adquieren nueva vida al ser iluminados, animados y transidos por el elemento asumente. El resultado de esta asunción es una nueva forma que determina la personalidad de cada nación (1950: X-XI).

Para Belaúnde el proceso asuntivo tiene la virtud de sintetizar, esto es, unir ambos elementos llegando a una conclusión en la cual se valoran y se confirman su influencia y su permanencia en la síntesis: "La oposición de tesis y antítesis aparece como una etapa posterior y es el comienzo de la escisión o desintegración. La vitalidad social se refleja en la supervivencia de la síntesis originaria", y esta síntesis originaria "corresponde a la naturaleza del hombre y de la realidad compleja de la vida" (Andrés Belaúnde, 1950: XI-XII). Podemos aceptar que en la historia latinoamericana los valores que se encuentran desde un lado y otro de las culturas que chocan, tanto materiales como espirituales, se relacionan siempre entrelazados sin perderse ninguno de sus elementos.

Remitidos entonces a la idea de asunción propuesta por Belaúnde vemos que estos valores espirituales se encarnan en el componente material del proceso, que son las personas. Es posible aceptar que en un primero momento no hay una trasposición de valores, sino que es un proceso histórico que respeta los pasos que el hombre mismo va dando, tanto desde la finalidad del conquistador de transmitir sus valores, como también la del conquistado de aceptar lo nuevo y compartir los suyos con el conquistador. No estamos hablando simplemente de ideas racionales, sino que referimos a valores: costumbres, creencias, prácticas, cosmovisiones, etc., que tienen que ver con el vivir mismo que luego conformarán la cosmovisión de la nueva cultura. Para llegar a que una cultura entera asuma valores espirituales que le son ajenos primero debe darse la asimilación de dichos valores por personas concretas, individuales, para luego ser asumido en las personas sociales.

Belaúnde afirma que los valores espirituales son asumidos en la misma naturaleza humana por su misma capacidad de existencia y porque el alma humana es capaz de recibir y asumir: "el alma humana es una forma no solo animante, sino asumente" (1950: XIV) Pero esta forma asumente es perceptible solamente en el acto de la asunción lo cual hace más compleja la existencia. Como la asunción se da en un proceso que puede durar años -y quizá sea un proceso sin fin- las personas de una cultura se verán enfrentadas a la continua experiencia asumente frente a los influjos de otra cultura, ya que es en el mismo contacto cuando se da la experiencia. De allí que las instituciones, los pueblos, las comunidades humanas sean un complejo proceso de asunción de estos 
valores espirituales que trascienden las diversas culturas y van conformando nuevas sociedades por la misma autonomía de los valores.

\section{El "proyecto asuntivo" de Leopoldo Zea.}

Leopoldo Zea nació en México en 1912 y fue uno de los más grandes filósofos de la historia latinoamericana. Estudió derecho y humanidades. Hacia 1939 conoce a José Gaos, que se convertirá en su maestro y quien lo ayudará en su carrera filosófica. Buscaba una América unida no sólo ideológicamente, sino a partir de la realidad, por la cual nuestros pueblos deben luchar. No aceptará, desde esta visión, que en América no exista un pensamiento propio o que el único pensamiento sea el del europeo. Escribió innumerables obras y muchas de ellas colaboran en el estudio de la historia del pensamiento latinoamericano.

Para Zea la dependencia de Latinoamérica ha pasado por dos etapas: la primera se remite a la Europa Ibera, que en la conquista de nuevas tierras buscaba la prolongación de su cultura; y la segunda, a la Europa occidental que buscaba la instauración de un nuevo orden. Esta dependencia ha generado un sentimiento de rechazo en los latinoamericanos que no han aceptado el pasado de sometimiento, para intentar hacer historia dejándolo de lado. Pero en este intento han rechazado el pasado colonial de la primera conquista, que intentó eliminar la cultura indígena, para asumir la pretensión de los libertadores occidentales, que no lograron librarse del todo de la dependencia: "una asunción que, por extraña e irrealizable, sólo implicará la aceptación de una nueva forma de dependencia" (Zea, 1977: 80). Frente a la negación de la existencia de una dependencia del colonialismo, en su lugar, se acepta una nueva colonización a partir de los países occidentales que asumirán el poder que el latinoamericano aún no alcanza. Es una nueva dependencia -dice Zea-, una forma libre de dependencia: libertad para elegir dependencia, pero quedando atrapado en ella.

Este rechazo de la dependencia viene dado por un complejo de una supuesta inferioridad ante la superioridad del europeo, que se ha encargado de convencer a los pueblos conquistados con sus ideas que sostienen esta teoría. Ante él, el hombre latinoamericano se ha creído a sí mismo como por debajo de la raza europea, perteneciendo a un pasado bárbaro que debe ser civilizado y educado según las normas del hombre superior. América fue considerada desde un primer momento como primitiva, donde sus indígenas eran seres que no habían evolucionado, por lo que era necesaria la conquista para que el status se superara. Esto aceptado así, crea en el conciencia de los lati- 
noamericanos el sentimiento de inferioridad ante el europeo que llega a estas tierras trayendo el progreso y proclamándose a sí mismo como quien trae la nueva humanidad.

No se quiere ser lo que se afirma que hemos sido como camino de resolución de lo que podemos llegar a ser. Este es un camino erróneo -según Zea- porque no se logra auténtica libertad intentando borrar todo lo vivido, con las consecuencias que han tenido, para intentar ser algo distinto de ello. Dice Zea: "la libertad era la negación del pasado hecho servidumbre; la civilización, la negación de la barbarie y el anacronismo de esa larga noche colonial. El afán por deshacerse a sí mismos como pueblos, implicaba la preocupación y el afán por ser un pueblo distinto" (1977: 113). De alguna manera el error llevó a caer en la imitación, e imitar implica seguir el camino de quien se acepta es superior, reconociendo la propia inferioridad. De esta manera se sigue dando una especie de subordinación a lo europeo, al cual hay que imitar en su pensar y proceder, para lograr entrar en el ámbito de un mundo superior.

En todo este recorrido que venimos haciendo nuevamente debemos hacer referencia necesaria a Hegel para poder comprender el proyecto asuntivo. Según Zea, en la dialéctica de Hegel, no existe la historia de América, pues, dentro del movimiento dialéctico, toda superación es al mismo tiempo negación y conservación. Si hablamos de la historia de Latinoamérica es simplemente como reflejo lejano del primer mundo, para lo cual solamente cuando ella entre dentro del proceso del espíritu absoluto, será asimilada a la historia, pero que pertenecerá al mundo europeo. Pero no olvidemos que para Hegel la realidad es racional, por eso es que en la conciencia histórica de América no se ha dado aún esta asunción, ya que las etapas por las cuales hemos atravesado son negadas lógicamente y no dialécticamente (en la realidad), por lo que esta asunción, en vez de contraponerse a la dialéctica, es parte constitutiva de ella. El proyecto asuntivo de Zea

tiene como punto de partida la propia realidad, por negativa que ella pueda parecer, para tratar de construir sobre ella y con ella, el mundo que se anhela. Negación, pero en sentido hegeliano, negación que es afirmación. Esto es, absorción, asunción de la propia realidad. Y dentro de la realidad, la historia, el pasado (1977: 134).

Desde esta no asimilación, del pasado histórico latinoamericano, es que se siguieron importando modelos extranjeros de cultura sin encontrar aún el valor de la propia por no aceptar el pasado. Por eso el proyecto asuntivo es tan necesario para que nuestros pueblos, en la conjugación de los elementos propios de estas tierras con los elementos extranjeros europeos, comprendan la conformación de su propia cultura. Por lo que la historia latinoamericana es posible desde una toma de conciencia a partir de la realidad en la cual estamos existiendo: 
el proyecto asuntivo se planteará entonces como una toma de conciencia sobre la realidad de la constitución histórica de la cultura latinoamericana, para desde allí saber lo que se es y proyectar lo que se puede llegar a ser. América Latina debe asumir su pasado para comprender el presente y proyectarse al futuro (Cruz, 2009: 73-84)

Resumiendo: para Zea, el proyecto asuntivo tiene que ver con asumir, que es absorber, en el sentido de aceptar y no rechazar, de integrar a lo propio y no de negar, de asimilar, no destruir. "Asimilar una función que ha de ser realizada por la propia realidad. Una realidad que ha de alimentarse de las expresiones de otras realidades, pero sin destruirse a sí misma en estos intentos" (1977: 137). En su proyecto de asunción se da la yuxtaposición de ambas culturas, en donde se debe combatir la idea de ser dos realidades diferentes: dos hombres, dos mentalidades, dos cosmovisiones, etc., para lograr hacernos de una sola idea de hombre y de una sola expresión de vida. Es en estas yuxtaposiciones que se absorben las unas a las otras, para lograr alcanzar la toma de conciencia necesaria para una historia que dé sentido al hombre latinoamericano a partir de la realidad. La identidad latinoamericana "posee ya una realidad que simplemente debe ser potenciada. No se trata de cambiar la realidad que esta América ha creado ya a lo largo de una historia llena de peripecias, dolores y sufrimiento, por una realidad que nada ha tenido que ver con ellos" (Zea, 1977: 138).

\section{Reflexión conclusiva: el ejemplo del Chaco boliviano.}

Para concluir este trabajo proponemos una mirada actual desde el ejemplo de la historia del Pueblo Guaraní del territorio del Chaco boliviano. En Bolivia la población indígena alcanza un gran porcentaje (más del $60 \%$ del total), conviviendo con una gran influencia histórica de población española desde la Conquista. Es allí donde hoy podemos constatar diversas prácticas culturales propias del hombre español (religiosas, devociones populares, modelos políticos y educativos), que conviven con un fuerte sentimiento de defensa autóctona por no perder los valores y prácticas propias de las culturas ancestrales (lenguaje, valores comunitarios, organización tribal, el lugar de los ancianos). Quizá sea Bolivia uno de los casos más extremos pero es allí donde la convivencia entre asunción y rechazo es muy visible.

Hacia el norte del país, en el altiplano, donde viven los descendientes de los Incas, "los coyas", se vive una gran rivalidad con el indio "guaraní" del sur chaqueño. Si bien esta rivalidad proviene desde antes de la conquista, el boliviano actual del norte tiende 
a asemejarse al modo de vida del hombre español occidental, y el guaraní intenta mantener sus propias tradiciones. Nos llama poderosamente la atención que en la historia del pueblo guaraní hay intentos de asunción de elementos extranjeros para aprovechar sus conocimientos de modo de ampliar sus dominios culturales, pero que luego estos intentos se vieron truncados por el afán de poder y la imposición del conquistador que, ya en la persona del criollo-mestizo, impuso sus ideas de manera violenta, alterando la posible síntesis ${ }^{4}$. Esto ha sido estudiado por historiadores como Isabelle Combes y Thierry Saignes, que sustentan el actual trabajo del antropólogo Elías Caurey ${ }^{5}$.

En primer lugar, a partir de esta constatación, nos surgen muchas preguntas en función de la propuesta de Belaúnde: ¿cómo lograr percibir en la realidad esta encarnación de valores, sin que en ello evitemos ver la negación que hay por parte de un gran porcentaje de habitantes latinoamericanos de origen indígena que, desde hace unos años, están esforzándose por recuperar sus costumbres ancestrales? Estamos de acuerdo con la forma asumente, propia del alma humana que propone Belaúnde, y que podemos confirmar en la misma vivencia de muchos latinoamericanos. Pero en este momento de la historia ¿no nos encontramos en una nueva situación? No creemos que haya en ello solamente una no aceptación, sino que creemos se trata de una necesaria autoafirmación de quienes somos, a partir de la protección de los valores culturales, espirituales y materiales, desde la experiencia que traemos desde que existimos sobre esta tierra.

Por otro lado, el proyecto asuntivo de Zea nos presenta que lo común en la historia latinoamericana es la negación en los diversos modos de asumir el mundo, sobre todo en los ámbitos de poder pues provienen de una mirada europea y es desde allí que se proponen las políticas en casi todos nuestros países. Es por eso que seguimos imitando, y se imita lo que se cree superior. En la época de la dictadura salvadoreña se buscaba alcanzar el estilo de vida de los Estados Unidos, algo que se confirma al estar en ese país: asumen claramente su condición indígena, pero pretenden vivir como otros: los yankees vistos como superiores. No se cree con la capacidad de poder alcanzar un modo de ser propio y que incluso puede ser ejemplo para aquellos países vecinos. Hay una negación del pasado histórico, que no busca absorber, sino rechazar e incluso eliminar el pasado de la conciencia social.

En un segundo momento, siguiendo a Zea desde el ejemplo citado, ¿estamos hablando de una negación del pasado? Cuando no se está imitando, ni tampoco rechazando,

\footnotetext{
${ }^{4}$ Chumiray, G., Apuntes para la historia del pueblo Guaraní de Bolivia, Teko Guaraní, Camiri, 2005, p. 14.

${ }^{5}$ Guaraní nacido en Ipitakuape (Cordillera boliviana), en 1977 es licenciado en sociología y en antropología y se dedica a la investigación de la cultura guaraní. La referencia la tomamos del libro Nación Guaraní, Una mirada a nuestro modo de ser, Territorio Guaraní, Bolivia, 2012.
} 
sino que las fuerzas se encaminan en cuidar y promover la propia cultura, como en el caso de los guaraníes ¿hay negación o rechazo de lo que somos? Creemos que esto se da sobre todo en los países donde ha alcanzado la mentalidad occidental, pero no podemos dejar de lado aquellos núcleos humanos que se han preservado a lo largo del continente y que parecieran estar al margen de lo que nosotros pensamos. Incluso podemos cuestionarnos si nuestra mirada desde la filosofía de la historia no está siendo presa de una cosmovisión occidental, propia de los que vivimos en países del Cono Sur.

Por otro lado, y siguiendo a Podetti, América es el continente del mayor mestizaje de la historia a partir de la gran confluencias étnicas y esto la coloca en un lugar de importancia mayor a la hora de la asunción de la característica principal de nuestras sociedades latinoamericanas que "forjan una identidad social y cultura nueva, fruto de varias sociedades multiseculares previas" (2008: 5). Desde este punto debe ser para nosotros un motivo de orgullo ser mestizos y no algo de lo que debamos deshacernos o rechazar. Esto fue lo que se intentó en un pasado: 'Lo 'mestizo', incorporado como una forma de conciencia, aparecerá como algo espurio, metido en el alma de todos los americanos y será lo que debe ser erradicado" (Roig, 2009: 289). La solución a esta situación que nos hace sufrir de inferioridad ante lo extranjero viene de la mano de aceptar quienes somos y, si somos el continente del mayor mestizaje, de alguna manera es hora de comenzar a aceptarlo para profundizar en nuestro mismo ser.

La síntesis viviente y el proyecto asuntivo colaboran en la toma de conciencia de que somos mezcla de culturas y que nuestro propio pensamiento es parte del proceso de pensamiento universal, pero que es producción del hombre latinoamericano que se plantea una filosofía desde la misma experiencia del ser latinoamericano. Pero aun siendo así, las grandes diferencias de modos de vivir y de pensar a lo largo de Latinoamérica hacen aún más compleja esta cuestión. Una necesidad, para el pensador latinoamericano, será adentrarse al interior de Latinoamérica, si es que quiere responder con coherencia a la síntesis viviente de Belaúnde. Será necesario un conocimiento del elemento material autóctono para poder reconocer la encarnación de los valores espirituales, como también necesitamos acercarnos físicamente al pasado -presente humana y materialmente hoy en varios lugares de Latinoamérica- para reconocer el proceso del proyecto asuntivo de Zea. 
Bibliografía

ARDAO, Arturo. (1987). La inteligencia Latinoamericana. Montevideo: Ed. Universidad de la República.

BELAúnde, V. Andrés. (1950) La síntesis viviente. Madrid: Ed. Cultura Hispánica.

BENAVidEs, L. Manuel (1994) Filosofía de la historia, Ed. Síntesis.

Castillo, Genara. (2007). El pensamiento filosófico de Victor Andrés Belaúnde. La noción de "Síntesis viviente". Mercurio Peruano, Revista de humanidades, 520, pp.28-45, Universidad de Pirhua. Versión digital en https://pirhua.udep.edu. pe/bitstream/handle-/11042/1614/Pensamientofilosofico_VABelaunde.pdf? sequence $=1$.

CAUREy, Elías. (2012). Nación guaraní. Una mirada a nuestro modo de ser. Territorio guaraní: Ed. Omar Rocha Velasco

Cruz, Manuel. (2008). Filosofía de la Historia. Madrid: Alianza.

CRUz, R., Edwin. (2009). La integración latinoamericana en el pensamiento de Leopoldo Zea. Revista Surmania, N³, págs. 73-84. Academia.edu.

Dussel, Enrique. (1992). El proyecto de una filosofía de la historia latinoamericana. UNAM, en http://www.enriquedussel.com/DVD\%200bras\%20Enrique\%20 Dussel/Textos/c/227.1992.pdf.

Chumiray, Guido. (2005). Apuntes para la historia del pueblo Guaraní de Bolivia. Camiri: Teko Guaraní.

LöwITH, Karl. (1998). El hombre en el centro de la historia. Barcelona: Herder.

Marías, Julián. (1979). Historia de la Filosofía, Madrid: Ed. Revista de Occidente.

Petit, M. Angélica. (1993). "El proyecto asuntivo en una solidaria y optimista dimensión universal”. Entrevista exclusiva a Leopoldo Zea. Cuadernos de Marcha, Año IX, Número 88, págs. 22-24.

Pieper, Josef. (1988). El Fin del Tiempo. Barcelona: Ed. Herder.

Podetti, J. Ramiro. (2008). Indigenismo, integración y política. Academia.edu.

PoDETti, J. Ramiro. (2007). Globalización y alteridad. Academia.edu.

Podetti, J. Ramiro. (2008). Asunción y síntesis de Víctor A. Belaúnde. Academia.edu.

Podetti, J. Ramiro. (2007). Cultura y alteridad: En torno al sentido de la experiencia latinoamericana. Caracas: Monte Ávila Editores.

Roig, Arturo. (2009). Teoría y crítica del pensamiento latinoamericano. Buenos Aires: Ed. Una ventana. 
Salazar, B. Augusto. (1965). Historia de las ideas en el Perú contemporáneo. Lima: Ed. Labor.

SANTiváñEz, V. Martín. (2014). Corrupción y regeneración: Víctor Andrés Belaúnde y "La Crisis presente". Revista de Indias, Vol. LXXIV, núm. 260, págs. 241-274

Zaffaroni, J. Carlos. (1966). Marxismo y cristianismo, Montevideo: Ed. Ap. O.C.E. ZEA, Leopoldo. (1969). Filosofía latinoamericana como filosofía sin más, México: Siglo XXI Editores.

ZEA. (1974). El pensamiento Latinoamericano. Versión digital en www.olimon.org/uan /americana.pdf.

ZEA. (1977). Filosofía de la Historia Latinoamericana. Versión digital en www.olimon.org /uan/americana.pdf. 